\title{
ON THE CATEGORIZATION OF HIGH ACTIVITY OBJECTS USING DIFFERENTIAL ATTRIBUTE PROFILES
}

\author{
M. Boldt ${ }^{1, *}$, A. Thiele ${ }^{1,2}$, K. Schulz ${ }^{1}$, S. Hinz ${ }^{2}$ \\ ${ }^{1}$ Fraunhofer Institute of Optronics, System Technologies and Image Exploitation IOSB, 76275 Ettlingen, Germany - \\ (markus.boldt, antje.thiele, karsten.schulz)@iosb.fraunhofer.de \\ ${ }^{2}$ Institute of Photogrammetry and Remote Sensing IPF, Karlsruhe Institute of Technology KIT, 76128 Karlsruhe, Germany - \\ (antje.thiele, stefan.hinz)@kit.edu
}

KEY WORDS: Synthetic Aperture Radar, SAR, TerraSAR-X, Time Series, Change Detection, Change Analysis, Clustering.

\begin{abstract}
:
Change detection represents a broad field of research being on demand for different applications (e.g. disaster management and land use / land cover monitoring). Since the detection itself only delivers information about location and date of the change event, it is limited against approaches dealing with the category, type, or class of the change objects. In contrast to classification, categorization denotes a feature-based clustering of entities (here: change objects) without using any class catalogue information. Therefore, the extraction of suitable features has to be performed leading to a clear distinction of the resulting clusters.

In previous work, a change analysis workflow has been accomplished, which comprises both the detection, the categorization, and the classification of so-called high activity change objects extracted from a TerraSAR-X time series dataset. With focus on the features used in this study, the morphological differential attribute profiles (DAPs) turned out to be very promising. It was shown, that the DAP were essential for the construction of the principal components.

In this paper, this circumstance is considered. Moreover, a change categorization based only on different and complementary DAP features is performed. An assessment concerning the best suitable features is given.
\end{abstract}

\section{INTRODUCTION}

The issue of change detection (CD) using remotely sensed imagery denotes a field of research being on demand for different applications. In case of detecting changes using time series data, the monitoring task is closely connected with $\mathrm{CD}$. For this, it is significant to consider data acquired regularly at similar time of day to produce meaningful detection results. Focusing on this restriction, the acquisition of imagery using SAR satellites hold important advantages against the optical alternative. SAR is an active sensing technique, illuminating the scenery with microwaves of a wavelength much longer than the visible part of the electromagnetic spectrum. Hence, it can be used independently from atmospheric effects and the time of day. Since 2007, the German SAR satellite TerraSAR-X (TSX) delivers imagery with a geometrical resolution of less than one meter by applying the High Resolution SpotLight mode HS300 (Airbus Defence and Space, 2014).

For CD based on SAR imagery, several approaches exist without extracting detailed information concerning the type or class of the detected changes (Lê et al., 2015; Su et al., 2014). One example for a comprehensive change analysis is given in (Weihing et al., 2010). There, the type of changes is determined with the help of a previously calculated land use / land cover (LULC) map of the investigated scene.

In general, the task of LULC classification is more or less limited by the reference and training data applied in a supervised or unsupervised processing scheme. To cope with these drawbacks and to propose a robust and easy to use method, an innovative strategy is described in (Boldt, 2018) which mainly bases on a categorization of the detected changes. As changes, so-called High Activity Objects (HAOs, compare Section 1.2) are considered. Each HAO is described by a feature vector comprising different complementary features. One such feature is given by the Morphological Differential Attribute Profiles (DAPs), representing an advancement of the concept of Morphological Profiles (see Section 2).

This study deals with the investigation of different attributes for the DAPs and their influence on a suitable HAO categorization result. In the following two subsections, the workflow for the extraction of the HAOs is outlined. The concept of the DAPs is shortly described in Section 2, including the attributes considered in this work. Results are shown in Section 3. The last Section 4 contains a concluding discussion of the study.

\subsection{Time Series Change Detection}

As input data, a time series consisting of 15 TSX HS300 amplitude images showing the scenery of Stuttgart (GER) airport and its surroundings is applied. For $\mathrm{CD}$, the well-known LogRatio (Dekker, 2005) method is utilized for each image pair in temporarily ascending order in combination with a variation of the unsupervised thresholding method of Rényi (Sahoo et al., 2004). A stack of 14 binary ChangeMaps results, containing the change objects (Connected Components, $\mathrm{CCs}$ ) at the foreground.

\subsection{High Activity Objects}

These binary maps are added leading to products called IndexMap (IM) and ActivityMap delivering information on the change frequency of imaged regions (Boldt, 2018). Basing on the IM, High Activity Areas (HAAs) can be identified which are characterized by the HAOs. HAOs are the CCs which were originally detected by the abrupt $\mathrm{CD}$ method and are located in

\footnotetext{
* Corresponding author
} 
the HAAs. Some real-world examples for HAAs are parking lots or construction sites.

\section{DIFFERENTIAL ATTRIBUTE PROFILES}

The DAP approach (Dalla Mura et al., 2010) can be seen as an advancement of the concept of Morphological Profiles (MPs) which was introduced at the beginning of the current century (Benediktsson et al., 2001). Especially relating on the task of SAR change categorization, the benefit of DAPs has already been described in (Boldt et al., 2013). In that work, significant characteristics and some theoretical backgrounds of the DAP approach are given. Consequently, such items are not part of this paper. The focus is set on the analysis of different attributes used to calculate the DAPs and on their impact on a suitable separation of change categories. For this, the well-known Principal Component Analysis (PCA) is applied.

First, a short overview on the basic concept of DAPs is given.

\subsection{Concept}

In contrast to MPs, DAPs base on the analysis of CCs contained in the specific image. Since no Structuring Element of fixed size and shape is considered, the DAPs denote a more flexible and precise way of image exploitation. Moreover, the DAP concept utilizes so-called attribute openings and closings (Boldt et al., 2013). This means that the CCs are analyzed by regarding their properties with respect to different, previously chosen attributes. For this, multiple attributes are available that are able to characterize the CCs in a complementary way (e.g. statistics, shape, or radiometry). For each attribute, a sequence of values has to be defined which is used as threshold criterion for the CCs detected in the image. According to the principle of attribute filtering, this means that each $\mathrm{CC}$ is checked against the given attribute value sequence leading to its retention or deletion (Boldt et al., 2013).

Subsequently, the attributes used in this study including their value sequences are explained.

\subsection{Attributes and Pre-Processing}

Different attributes are considered in this study which are outlined in the following. For each attribute, a sequence of five values is defined to model each HAO, leading to a 10dimensional DAP vector. The sequence intervals are subdivided into four equally spaced divisions.

\subsubsection{SAR-Specific Attributes}

Two SAR-specific attributes are chosen. The first is given by the Coefficient of variation (Cov), denoting a measure of local homogeneity in the amplitude image, calculated by the ratio of standard deviation and mean in a moving window. The second one represents the normalized radar cross section, given by the Sigma Nought (Sig0) coefficient scaled to decibels [dB].

Concerning the choice of a value sequence for the Cov attribute, we refer to (Schulz et al., 2012), where suitable thresholds for Cov image layers are extracted in a simulation-based way, leading to a reasonable scaling of the image content. Here, the explained scaling interval is adapted to characterize the local homogeneity of HAOs. The sequence $\chi^{C o v}$ is given with:

$$
\chi^{\operatorname{Cov}}=[0 ; 0.5 ; 1 ; 1.5 ; 2],
$$

consisting of five sequence values.

For the attribute sequence of the second SAR attribute, Sig0, a maximum value of $+10 \mathrm{~dB}$ was chosen according to (Klausing et al., 2000). Focusing on the minimum edge of the interval, the sensor-specific value representing the background noise of the system can be applied. With different backscatter analyses of asphalt surfaces, it was found that a value of $-22 \mathrm{~dB}$ can be applied as an adequate threshold, coinciding with the Noise Equivalent Sigma Zero of current X- and C-Band SAR sensors (Schulz et al., 2012). Consequently, the value sequence is defined with:

$$
\chi^{\operatorname{Sig} 0}=[-22 ;-14 ;-6 ; 2 ; 10][d B] .
$$

\subsubsection{Shape-Specific Attributes}

Six shape parameters are considered in total: Moment of inertia (Moi), area (Ar), the diagonal of the bounding box of the HAO (Diag), roundness (Round), compactness (Comp), and density (Dens).

The Moi is the so-called Hu-moment of first order $(\mathrm{Hu}, 1962)$, which can be interpreted as mass moment of inertia, representing the extension of a $\mathrm{CC}$ according to its centroid or center of mass. Hence, the centroid of a $\mathrm{CC}$ can be seen as origin for a rotation axis and its gray values can be taken as density values. The Humoment of first order is defined as (Huang et al., 2010):

$$
M o i=n_{20}+n_{02},
$$

with the scale invariants

$$
n_{i j}=\frac{m_{i j}}{m_{00}^{\left(1+\frac{i+j}{2}\right)}},
$$

and the central moments

$$
m_{i j}=\sum_{x=1}^{P} \sum_{y=1}^{P}(x-\bar{x})^{i}(y-\bar{y})^{j} I(x, y),
$$

with the image coordinates $x$ and $y$ of the current HAO, the HAO centroid coordinates $(\bar{x}, \bar{y})$, the count of HAO pixels $P$, and the gray value $I(x, y)$ of the input image $I$.

With these formulas, it is observable that line-shaped HAOs produce higher Moi values than more compact HAOs. Moreover, concerning the HAO gray values, constantly high gray values lead to relatively high Moi values. Useful attribute values can be extracted by considering the shape and radiometry of HAOs. For example, a rectangular HAO with edge lengths 10 and 40 pixels lead to a Moi of approximately 400 (HAO with constant bright gray level, $I(x, y)=255, \forall x, y \in I)$. If this HAO is converted into a line-shaped structure, the Moi gets higher (HAO with edge lengths 8 and 50 pixels: $M o i \approx 700$ ). From these considerations, the value sequence is extracted to be:

$$
\chi^{M o i}=[0 ; 200 ; 400 ; 600 ; 800] .
$$

Focusing on the Ar attribute, representing the count of pixels of each HAO, the value sequence is deduced from the scope of our change analysis, which aims on the detection of small-sized objects like vehicles or smaller construction sites. In general, it should be kept in mind that the count of pixels of such objects always depends on the pixel size of the available image data. Here, the sequence is chosen with:

$$
\chi^{A r}=[8 ; 106 ; 204 ; 302 ; 400] .
$$

From the Ar attribute sequence, the length of the diagonal of the bounding box surrounding the HAO can be easily derived. The maximum area of 400 pixels can be exemplary arranged in a $\mathrm{HAO}$ with the edge lengths 133 and 3 leading to a very elongated shape. For this rather theoretical HAO representation, a Diag value of 133 (pixels) results. The minimum size of a HAO can be 
modeled by a Diag length of 5 pixels. From this, the sequence results as:

$$
\chi^{\text {Diag }}=[5 ; 28.75 ; 52.5 ; 76.25 ; 100] .
$$

Another shape attribute is given by the roundness parameter Round, which serves to evaluate the similarity of a HAO with its minimal approximating ellipse. It is defined with:

$$
\text { Round }=\frac{\Delta\left(f o c_{A}, f o c_{B}\right)}{l_{m a}},
$$

with $\Delta()$ being the distance function to calculate the distance between the two focal points $f o c_{A}$ and $f o c_{B}$ of the ellipse, and with the length $l_{m a}$ of the major axis.

Resulting values for Round cover the interval of [0;1], where 1 stands for a HAO being ideally line-shaped. Consequently, Round is 0 when the HAO is a circle. With this, the value sequence can be written as:

$$
\chi^{\text {Round }}=[0 ; 0.25 ; 0.5 ; 0.75 ; 1] .
$$

The Comp parameter represents a measure to evaluate the similarity of a HAO to a surrounding, minimal approximating rectangle:

$$
\operatorname{Comp}=\frac{l_{\text {rect }} w_{\text {rect }}}{s_{H A O}},
$$

With the length $l_{\text {rect }}$, width $w_{\text {rect }}$ and size $s_{\text {rect }}$ of the rectangle. It can be seen that a Comp value of 1 occurs in case of an ideally compact or rectangularly-shaped HAO. Nevertheless, even higher values are possible, which motivates the extraction of a suitable value sequence by using test results derived from a complementary available dataset. As upper bound of the sequence, the maximum value of this test was taken and a tolerance of twice the standard deviation was added. Finally, the sequence for the Comp attribute is formulated as follows:

$$
\chi^{\operatorname{Comp}}=[0 ; 1.75 ; 3.5 ; 5.25 ; 7] .
$$

In contrast to roundness and compactness, the last shape parameter, density (Dens), serves to evaluate the spatial distribution of HAO pixels. A HAO is ideally dense, when its shape is quadratic. Otherwise, a HAO is not dense when it is lineshaped (Boldt, 2018). The Dens attribute is calculated with:

$$
\text { Dens }=\frac{\sqrt{s_{H A O}}}{1+d_{e}}
$$

with the diameter $d_{e}$ of a surrounding ellipse:

$$
d_{e}=\frac{\sqrt{s_{H A O}}}{2 \sqrt{a^{3} b^{2} /\left(b^{2} \cos ^{2} \omega+a^{2} \sin ^{2} \omega\right)}},
$$

where $a$ stands for half the major axis length, $b$ for half the minor axis length, and $\omega$ for the angle between the horizontal axis of the image and the major axis of the ellipse.

Since the actual Dens value depends on the size and shape of the current HAO, a generally valid sequence interval does not exist. A low value near 0 represents a not-dense HAO. Analogously to the Comp attribute, a suitable value sequence was calculated by tests, leading to:

$$
\chi^{\text {Dens }}=[0 ; 7.125 ; 14.25 ; 21.375 ; 28.5]
$$

\subsubsection{Feature Matrix}

With the descriptions given above, it follows that for each HAO, a feature vector consisting of DAP values for eight attributes (shape- and SAR-specific) is calculated. Since a sequence of five values was chosen for each attribute, 10-dimensional DAP vectors were extracted. Therefore, the dimension of the HAO feature vectors is 80 . This fact combined with the high amount of HAOs $(34,363)$ leads to a high-dimensional feature matrix, which has to be reduced to avoid problems such as the so-called curse of dimensionality (Hsu, 2007).

This reduction is performed using the PCA, containing the benefit of analyzing the impact of the original features on the resulting principal components (PCs). This analysis can be accomplished by evaluating the loadings of the linear combinations describing the PCs (Boldt, 2018). In this way, focusing on a suitable separation of different change categories, an optimal feature or combination of features can be identified. With respect to the feature matrix available in this study, 11 PCs are found which represent $81.4 \%$ of the total data variance (Figure 1). From this, the first two PCs already hold about $44 \%$.

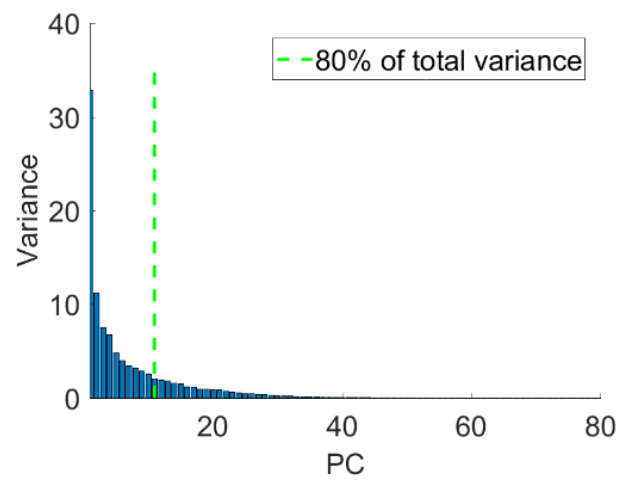

Figure 1. Eigenspectrum showing the total variance for each PC (in [\%]). The dotted green line marks the first $11 \mathrm{PCs}$.

With the evaluation of the loadings for the $11 \mathrm{PCs}$, it can be concluded, that the SAR-specific (Cov, Sig0) and the roundness (Round) DAP attributes have the biggest influence on the reduced feature space representation (Figure 2).

In Figure 2 (A), the loadings of the input features for the first PC are visualized. To differentiate between features with low and high influence on the PC, a dotted line is plotted representing $75 \%$ of the biggest loading value. With this, most significant features are identified for each of the 11 PCs. A histogram results (Figure 2, B) which contains the frequencies for the features, showing how often they exceed the $75 \%$ border. It can be observed that in contrast to the other DAP attribute groups, the Cov, Sig0, and Round attributes have the highest frequency of most significant features. From this it follows that the detected HAOs are well-separable by considering these attributes. Furthermore, the Round attribute represents the most sensible shape parameter. Hence, it can be stated that the detected smallscaled HAOs show a high variance with respect to this feature. For other scaled change objects, other shape feature might be more important. This should be kept in mind when adapting the method to other change analysis tasks.

In summary and with focus on the categorization of HAOs, this attribute combination leads to a suitable change differentiation. Therefore, it might be sufficient to reduce the high-dimensional HAO feature vector only on these features. This analysis can be part of further studies. 
A

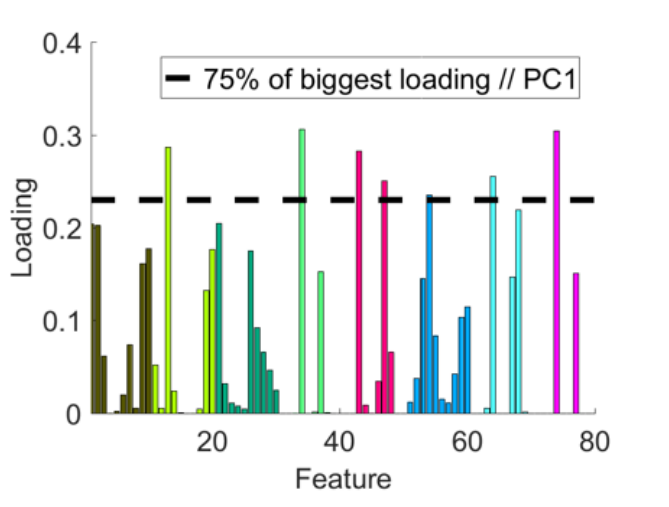

B

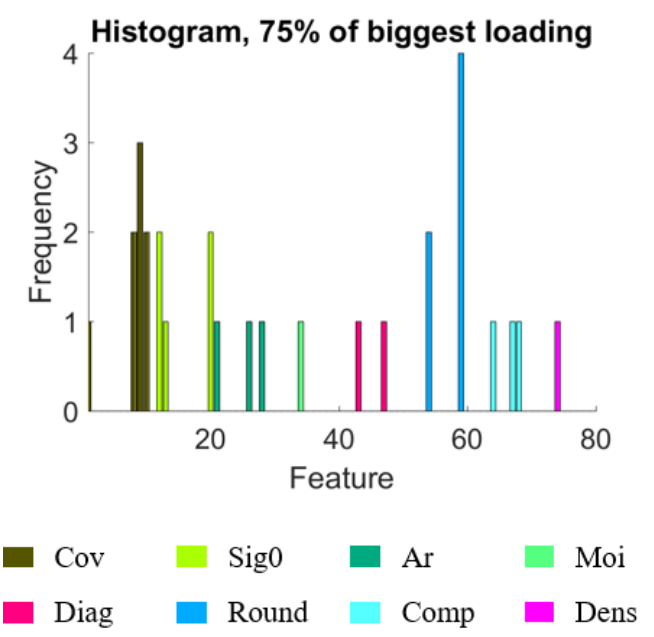

Figure 2. Loadings of first PC (A). Dotted black line symbolizes a threshold (here: $75 \%$ of biggest loading) to identify most significant features. B: Histogram of most significant features over all 11 PCs.

\section{CATEGORIZATION OF HIGH ACTIVITY OBJECTS}

Based on the reduced feature matrix, the HAOs are categorized by applying an unsupervised configuration of the commonly known k-means clustering algorithm. For this, the optimally matching number of categories was calculated by evaluating the mean quantization error as described in (Boldt et al., 2014). Five categories were found to which the HAOs were assigned. Figure 3 contains the categorization result, illustrated in three different HAAs.

The first HAA denotes an industrial area, consisting of different parking lots for cars and trucks and of collecting or loading / unloading points (Figure 3, A). The second HAA shows a subset of the airport area, containing surrounding structures and parts of the airport ramp (Figure 3, B). The third HAA represents some suburban structures with business complexes close to the airport ramp, including airplane parking lots. Moreover, this HAA contains a construction site, which was on progress during the time series data acquisition (Figure 3, c.1 to c.2).

With respect to the categorization results, it can be seen that semantically different subsets contain different change categories dominating these areas. For example, construction sites are dominated by the green category (Figure 3, A.3, a; B.3, a, b), whereas car parking lots are composed by the blue and red categories (Figure 3, A.3, b; B.3, C; and partially C.3, b). In contrast, airplane parking lots at the airport ramp show a dominance of the magenta-colored category (Figure 3, B.3 lower half and C.3, a).

The subset C. $3 \mathrm{~b}$ of Figure 3 represents an interesting area which is suitable to show the precision of our method. As already mentioned, this area denotes a construction site, which was in progress during the acquisition period of the time series. The observable fact that a small construction site category (green) was found besides the car parking lot categories (blue and red) leads to the assumption that the construction site was partially finished during the investigated period. The detected construction site category at the upper right edge could exemplary be caused by a local aggregation of soil or debris.

In summary, it can be resumed that the chosen DAP attributes enable a precise detection of change categories having different semantical meaning.

\section{CONCLUSION}

In this study, the concept of Differential Attribute Profiles (DAPs) was used in connection with the task of change categorization in high resolution SAR time series data. As changes, so-called High Activity Objects (HAOs) are considered, characterizing very frequently changing areas of the investigated scenery (e.g. parking lots and construction sites).

Several complementary attributes are used for the HAO-based DAP calculation. In detail, eight different attributes consisting of the two main divisions SAR-specific (Coefficient of variation, normalized radar cross section Sigma Nought ) and shape (area, moment of inertia, diagonal of bounding box, roundness, compactness, and density) were considered. For each attribute, a value sequence of five values was applied, leading to a 34,363 x 80 sized feature matrix (34,363 detected HAOs).

To avoid problems such as the curse of dimensionality, this highdimensional feature matrix was reduced by using the well-known Principal Component Analysis (PCA), offering the opportunity to analyze the impact of the original features on the resulting principal components (PCs). This is accomplished with the help of the so-called loadings, which represent the coefficients of the input features in the linear combinations of the resulting PCs. It was observed that the SAR-specific attributes and the roundness feature are most significant for a differentiation of the HAOs.

For the final categorization step, the first 11 PCs were taken which comprise approximately $80 \%$ of the total data variance. The reduced, 34,363 x 11 feature matrix was taken as input for an unsupervised version of the k-means algorithm, leading to the calculation of the optimal number of clusters. Five change categories were found in total. For the evaluation of the existing local semantics, the HAOs were color-coded matching to their categories and visualized in the time series mean amplitude image.

The robustness and precision of the categorization results were evaluated in three representative High Activity Areas (HAAs). It was observed that construction sites, car and airplane parking lots contain a characterizing dominance of different categories. Hence, these land use classes can be easily identified. The precision of this identification was shown with the example of a construction site which was partially in progress during the investigated period.

It was shown that the presented attributes for the calculation of the DAPs are well-suitable for a capable differentiation of change categories. Furthermore, it was observed that especially the SARspecific features combined with the roundness attribute lead to promising results when focusing on small-sized change objects. Generally, it should be kept in mind that for other change object sizes, other attribute combinations might be more meaningful. 
Moreover, the DAP method enables the usage of nearly all possible attributes to model the image content in a complementary way.

Consequently, future studies might deal with the analysis of other attributes which are suitable for the task of change categorization. Another interesting aspect to deal with is given by the detection of change objects of different scale and their correlation with certain DAP attributes. Hence, suitable attributes for different scopes of change detection (e.g. monitoring of urban, rural or forest) can be proposed.

\section{REFERENCES}

Airbus Defence and Space, Geo-Intelligence Programme Line, 2014. TerraSAR-X Image Product Guide, Basic and Enhanced Radar Satellite Imagery. URL (accessed on 2018/03/28): https://mdacorporation.com/docs/default-source/product-specsheets/geospatial-services/image_product_guide.pdf, 24 pages.

Lê, T. T., Atto, A. M., Trouvé, E., 2015. Change Analysis using Multitemporal Sentinel-1 SAR Images. In: Proceedings of the IEEE International Geoscience and Remote Sensing Symposium (IGARSS), pp. 4145-4148.

Su, X., Deledalle, C.-A., Tupin, F., Sun, H., 2014. Change Detection and Classification of Multi-Temporal SAR Series based on Generalized Likelihood Ratio Comparing-andRecognizing. In: Proceedings of the IEEE International Geoscience and Remote Sensing Symposium (IGARSS), pp. 1433-1436.

Weihing, D., Poncet, F. v., Schlund, M., Lang, O., 2010. Change Analysis with TerraSAR-X Data. In: Proceedings of the ISPRS TC VII Symposium IAPRS, Vol. XXXVIII, Part 7B, pp. 644-647.

Boldt, M., 2018. Änderungsanalyse in Zeitreihen hochaufgelöster SAR-Satellitenbilder. PhD thesis, XIV, 188 pages, DOI: 10.5445/IR/1000079162.

Dekker, R. J., 2005. SAR change detection techniques and applications. In: Proceedings of the 25 th EARSeL Symposium; Global Developments in Environmental Earth Observation from Space, pp. 63-69.

Sahoo, P. K., Arora, G., 2004. A thresholding method based on two-dimensional Renyi's entropy. In: Pattern Recognition, Vol. 37, No. 6, pp. 1149-1161.

Dalla Mura, M., Benediktsson, J. A., Waske, B., Bruzzone, L., 2010. Morphological Attribute Profiles for the Analysis of Very
High Resolution Images. In: IEEE Transactions on Geoscience and Remote Sensing, Vol. 48, pp. 3747-3762.

Benediktsson, J. A., Arnason, K., Pesaresi, M., 2001. The Use of Morphological Profiles in Classification of Data from Urban Areas. In: Proceedings of Remote Sensing and Data Fusion over Urban Areas, IEEE/ISPRS Joint Workshop, pp. 30-34.

Boldt, M., Schulz, K., Thiele, A., Hinz, S., 2013. Using Morphological Differential Attribute Profiles for Change Categorization in High Resolution SAR Images. In: Proceedings of the International Archives of the Photogrammetry, Remote Sensing and Spatial Information Sciences, ISPRS Hannover Workshop, Vol. XL-1/W1, pp. 29-34.

Schulz, K., Boldt, M., Even, M., 2012. Generalization of the CoVAmCoh Analysis for the Interpretation of Arbitrary InSAR Images. In: Proceedings of the IEEE International Geoscience and Remote Sensing Symposium (IGARSS), pp. 7444-7447.

Klausing, H., Holpp, W., 2000. Radar mit realer und synthetischer Apertur, Konzeption und Realisierung. Oldenbourg-Verlag München Wien, ISBN: 3486234757, 399 pages.

Hu, M.-K., 1962. Visual Pattern Recognition by Moment Invariants. In: IRE Transactions on Information Theory, Vol. 8, pp. 179-187.

Huang, Z., Leng, J., 2010. Analysis of Hu's Moment Invariants on Image Scaling and Rotation. In: Proceedings of the $2^{\text {nd }}$ International Conference on Computer Engineering and Technology (ICCET), pp. 476-480.

Hsu, P.-H., 2007. Feature Extraction of hyperspectral images using wavelet and matching pursuit. In: ISPRS Journal of Photogrammetry and Remote Sensing, Vol. 62, pp. 78-92.

Boldt, M., Thiele, A., Schulz, K., Hinz, S., 2014. Unsupervised Segmentation of HR SAR Time Series Amplitude Imagery Aiming on Context Based Change Categorization. In: Proceedings of the $10^{\text {th }}$ European Conference on Synthetic Aperture Radar (EUSAR), pp. 616-619. 

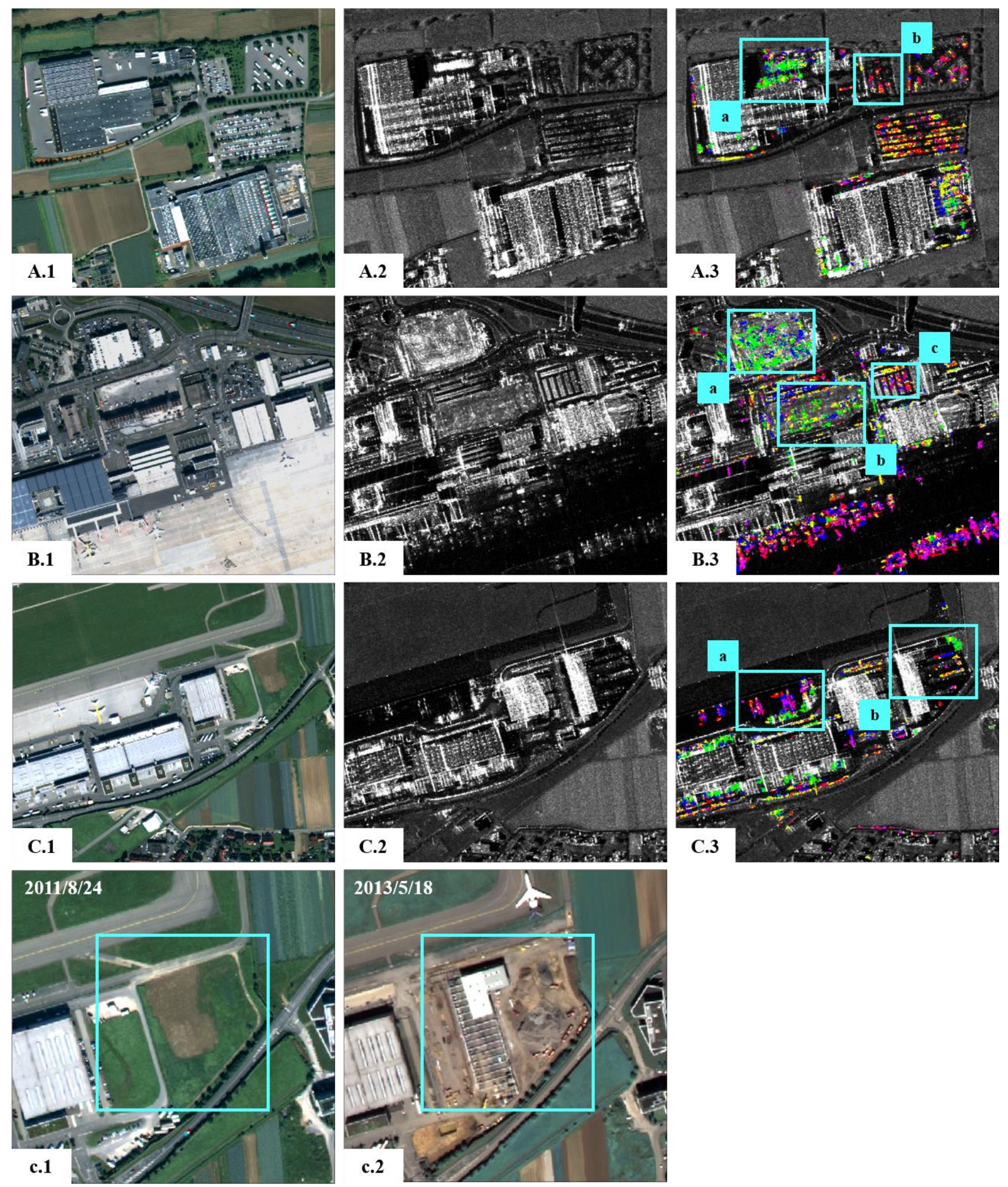

Figure 3. Results of change categorization illustrated by three exemplary HAAs (A to C) representing an industrial area (A), surrounding structures of airport area and subset of airport ramp (B) and some business complexes nearby the airport ramp (C). 1 (A to C): WorldView-2 image, 2 (A to C): Time series mean TSX amplitude image, 3 (A to C): Image of 2 with overlaid color-coded

HAOs, where each color stands for a specific change category. c: WorldView-2 images representing a detailed view on the construction site in C.3, b, detected by the method. Cyan-colored rectangles highlight AOIs: Car parking lots (A.3, b; B.3, c), loading

/ unloading and collecting points (A3.a), construction sites (B.3, a; B.3, b), airplane parking lots (C.3, a) and a mixture of a construction site and an already finished car parking lot $(\mathrm{C} .3, \mathrm{~b})$. 